\title{
THE RECORDING OF NERVE AGTION POTENTIALS THROUGH SKIN IN MAN
}

\author{
BY \\ G. D. DAWSON and J. W. SCOTT \\ From the National Hospital, Queen Square, London
}

\section{Introduction}

Electrical stimulation of peripheral nerve in man has been shown to produce cerebral responses (Dawson, 1947), and analysis of these responses has made clear the need for some means of investigating the nature of the sensory afferent volleys concerned. Experiments carried out in 1945 suggested that it was sometimes possible to record through the skin action potentials in the ulnar nerve of one of us (G.D.D.) when the nerve was stimulated electrically at the wrist. These observations were later repeated independently for the median nerve by Dr. Merton, but on both occasions the action potentials were small and could not be applied to the problems in hand ; the experiments were therefore not carried further. However it later seemed worth while to repeat these experiments to see if better results could be obtained. The only previous reports of the possibility of recording through skin nerve action potentials in man which have been found are those of Rusinov and Chugunov (1939, 1940) and Rusinov (1943, 1947). They report recording nerve action potentials from over the median and ulnar nerves above the elbow when | they stimulated proprioceptive sensory endings by hanging weights on the fingers, and when they pricked the hand or brushed the skin of the fingers. We have repeated the experiments of these authors with tactile and painful stimuli, but we have been unable to obtain any records resembling theirs so long as the muscles near to the recording electrodes were well relaxed. We have not repeated the experiments in which muscles were stretched, as it is difficult to avoid contraction of muscles with the stimuli they used : up to $500 \mathrm{~g}$. hung on one finger. They do not seem to have carried out adequate control experiments to exclude the possibility that they were recording muscle action potentials. From the experiments to be described it now seems clear that, after electrical stimulation at the wrist, action potentials of useful size may be recorded from the skin over the more superficial parts of the median and ulnar nerves near the elbow. Since it is not generally known that this is the case, and since the technique appears to have application to the study of peripheral nerve injury and disease, it is the purpose of this paper to report the methods used and to describe the characters of the action potentials which may be recorded.

\section{Methods}

The course above the elbow of the superficial part of the median or ulnar nerve to be examined was plotted, using an electrical stimulus, and was marked on the skin. A pad electrode $1.5 \mathrm{~cm}$. in diameter on a handle was used as a cathode to search for the places at which the nerve was most easily stimulated, and the stimulator anode was attached to a plate electrode $3.5 \mathrm{~cm}$. by $6 \mathrm{~cm}$. placed in any convenient position on the arm. To reduce the spread of the stimulus, and to give more precise location of the nerve, a small shock was used, just great enough to cause visible twitching of the forearm and hand muscles. The length of nerve which was relatively superficial varied considerably from one subject to another. Sometimes a length of more than $6 \mathrm{~cm}$. was found over which the threshold for stimulation altered little; sometimes the length was less than $3 \mathrm{~cm}$. In the same way the most superficial part of the nerve was plotted at the wrist. The skin over the nerve trunk was then rubbed with Cambridge electrode jelly to produce an erythema and afterwards washed with soap and water. This is important, as the production of an erythema reduces the skin resistance and the trouble from stimulus escape, but this gain may be lost if an excess of electrode jelly is left on the arm. The recording electrodes, with a small quantity of electrode jelly on each, were then applied to the skin over the nerve above the elbow and the stimulating electrodes at the wrist. In order to obtain good results the interelectrode resistance, measured with direct current at not more then 1 volt, should be less than 10,000 ohms, but it is advisable not to prick or abrade the skin under the stimulating electrodes in order to reduce the resistance, as this may make the stimulus painful at the site of stimulation and prevent muscular relaxation. Records free from muscle action potentials were obtained only when the muscles near to the recording electrodes were relaxed. For the same reason the stimulus was always 


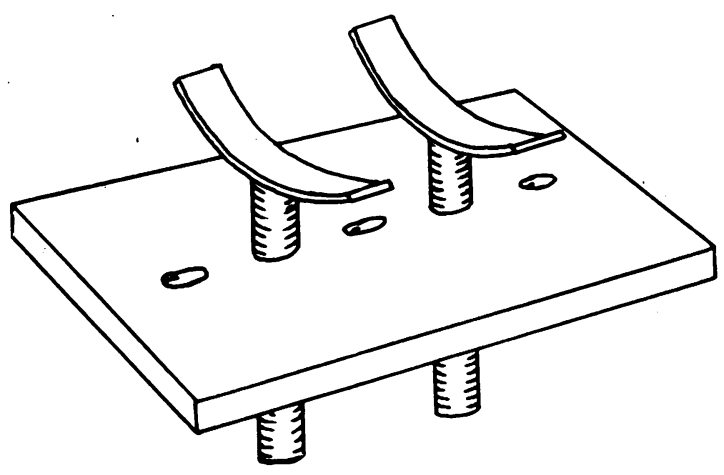

FIG. 1.-The diagram shows the form of the recording electrodes before padding with lint. The distance between the holes in the bakelite supporting block is $1 \mathrm{~cm}$. Blocks of different lengths were used for different numbers of electrodes.

applied at the wrist and the record made from over the nerve above the elbow. In this way the only muscles excited by the shock were those in the hand, which were as far as possible from the recording electrodes.

The recording electrodes which were used were of the form shown in Fig. 1, narrow rectangles of silver sheet $1 \mathrm{~mm}$. thick, $0.5 \mathrm{~cm}$. wide, $2.5 \mathrm{~cm}$. long, and curved in their long axes to locate the nerve under them. They were mounted at their centres on threaded silver tubes and covered with lint and gauze pads soaked in brine. The silver tube stems were screwed into a bakelite block in which there was a line of holes at intervals of $1 \mathrm{~cm}$. ; for recording nerve action potentials two or three electrodes were used at intervals of $3 \mathrm{~cm}$. in a block longer than that shown in Fig. 1. The electrodes were arranged with their long axes parallel, and the blocks were pressed on to the skin by rubber or webbing straps with the length of the electrodes as nearly as possible at right angles across the course of the nerve. The form of the electrodes is important as they must be small in the direction along the length of the nerve if the site of pick-up is to be even approximately known. The effect of movement of the electrodes in a direction across the length of the nerve is minimized if they are made large in this direction ; a movement of a small circular electrode away from the nerve by only a centimetre may lose the action potentials. For recording action potentials in the thenar or hypothenar muscles, surface electrodes were used, placed over the bellies of the muscles. The electrodes were either of the strip form used for recording the nerve action potentials, or were silver cups, $1 \mathrm{~cm}$. in diameter, held on with collodion.

Several arrangements of stimulating electrodes have been used. In early experiments a large anode was placed on the hand or arm and for the cathode a small pad electrode was placed over the nerve; the electrodes were of the same type as those used for plotting the course of the nerve. Later it was found that the stimulus escape could be greatly reduced if both electrodes were made small and of equal size and were placed close together. When only the nerve action potentials proximal to the point of stimulation were being recorded, a pair of electrodes of the same type as those shown in Fig. 1, $\underset{\mathbb{D}}{Z}$ with the more proximal one the cathode and the more distal one the anode, was found to be suitable. However, this arrangement of the stimulating electrodes, with the anode over the nerve distal to the cathode, may lead to blocking of some of the fibres conducting impulses to the periphery, and the volleys travelling away from the electrodes in the two directions will not be $\overline{\text { ?. }}$. comparable. To avoid this difficulty, when action $\stackrel{2}{F}$ potentials in muscles distal to the point of stimulation - . were being recorded, as well as nerve action potentials $\overrightarrow{\vec{F}}$ proximal to it, small silver cup electrodes were used. $\stackrel{\oplus}{+}$ One of these over the nerve was made the cathode ; and the other, placed several centimetres medially or laterally, was made the anode. The stimulating voltage used had $\frac{\bar{\omega}}{\frac{\sigma}{\sigma}}$ a steep front rising to its peak in not more than $4 \mu \mathrm{sec}$.,$\stackrel{\mathbb{Q}}{\varrho}$ and an exponential delay, usually with a time constant of $50 \mu \mathrm{sec}$. The stimulating electrodes were both un- $\infty$ earthed and were coupled to the stimulator through a $\vec{\circ}$ Muirhead Wide-Range transformer. When working between input and output circuits of $2,000 \mathrm{ohms}$ im- $\vec{\omega}$ pedance this transformer has a flat frequency response? up to 150 kilocycles per second. Since the stimulator has a low output impedance the form of the stimulating. current in the tissues did not follow the form of the $\vec{N}$ potential difference between the stimulating electrodes. + However, the results obtained with this stimulator wetein consistent, and it has been pointed out (Walterco and Ritchie, 1945) that a stimulator with a low outpht음

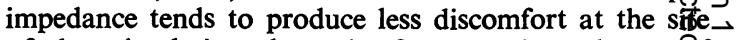
of the stimulating electrode, for any given degree $8 f z$ nerve stimulation, than one of high output impedanee, and for this reason the low impedance type was preferreg. This is important not only when examining patients also when doing laboratory experiments on volunteę⿸厂二⿺乚一匕 since the subject must be as relaxed and comfortable possible if the records are to be free from muscle action potentials. Occasionally when a search was being made ${ }^{\bullet}$ for action potentials from fibres with higher thresholds a shock of the same form but with a time constant of 0.4 msec. was used.

The recording amplifiers, which have balanced input circuits, were used without grid resistances in the input $\frac{}{\Phi}$ stages. An earth connexion was made to the subject's forearm, between the electrodes used for stimulating and $\overline{\bar{O}}$ those used for recording the nerve action potentials. $\frac{3}{3}$ Differences in the contact resistance at the recording electrodes may produce a serious imbalance in the input? circuits if grid resistors are used, much greater than the imbalance of the amplifiers themselves. This imbalance? may increase seriously the stimulus artefact in the records, and it is easier to reduce this effect if the grid 3 resistors are omitted. The frequency response of the amplifiers was set to be uniform within 10 per cent.윽 from $20 \mathrm{c} / \mathrm{s}$. to $700 \mathrm{c} / \mathrm{s}$., and to attentuate by half frequencies of $5 \mathrm{c} / \mathrm{s}$. and $2,800 \mathrm{c} / \mathrm{s}$. The response of one음 amplifier and recorder to a rectangular pulse with $a_{\supset}$ duration of $2 \mathrm{msec}$. and an amplitude of $20 \mu \mathrm{V}$., from a음 source of 500 ohms impedance, is shown in Fig. 2c. Fifty records were superimposed, and the peak-to-peak $\mathrm{N}$ amplitude of the inherent irregularity of the apparatus $\mathrm{N}$ (" noise") is about $4 \mu \mathrm{V}$. The output of the amplifiers 


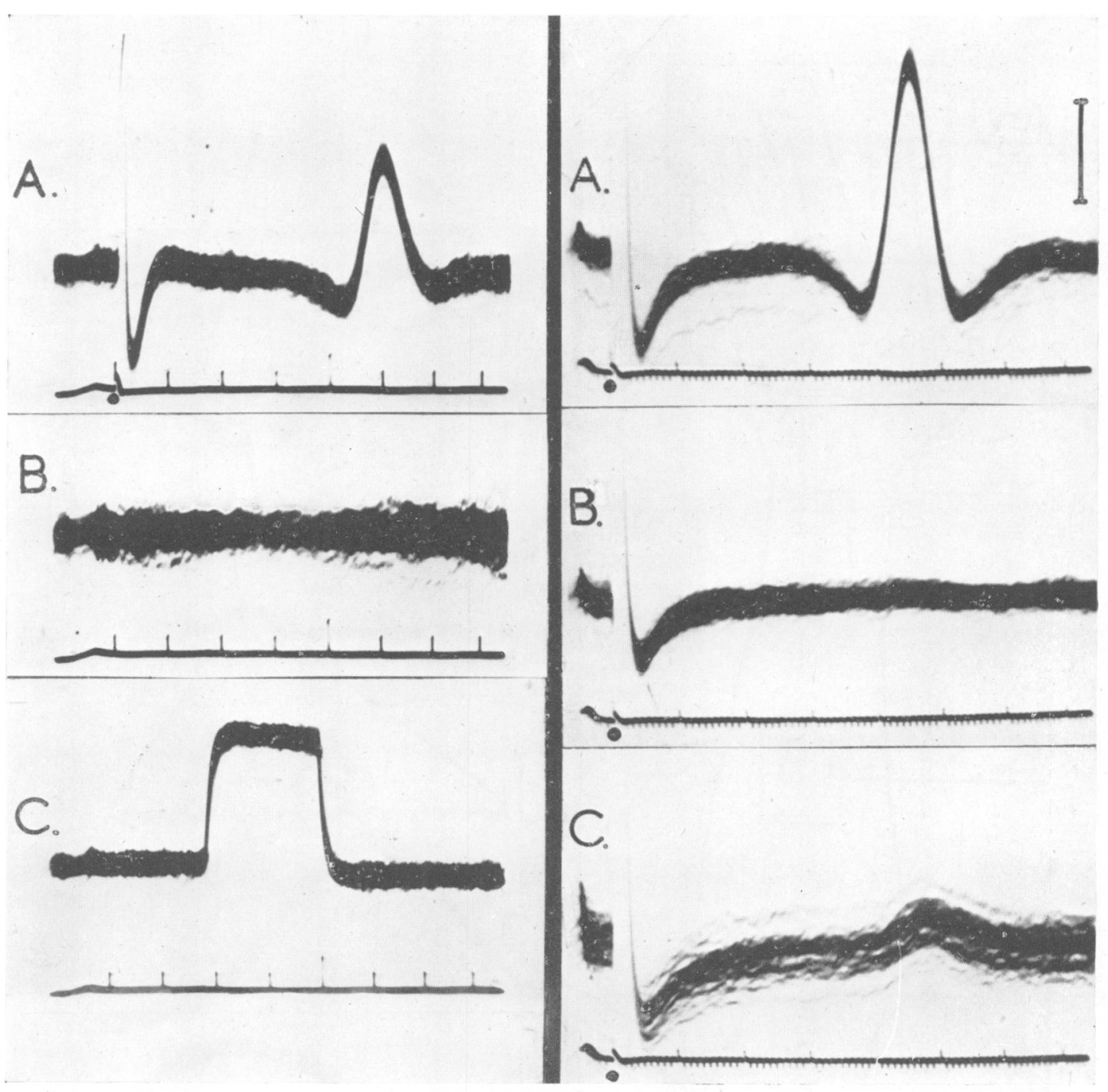

FIG. 2 (A, B, C, left).-The records show the potential differences between a pair of electrodes on the skin over the median nerve above the elbow. In (A) electrical stimuli were applied to the nerve at the wrist at the point indicated by the black dot below the time scale. In (B) the conditions were identical except that no stimuli were applied. Record (C) shows the response of the recording system to a rectangular pulse of $20 \mu \mathrm{V}$. amplitude delivered from a source of $500 \mathrm{ohms}$ impedance. The time scales show intervals of $1 \mathrm{msec}$. In these and in all succeeding records fifty traces were superimposed.

FIG. 3 (A, B, C, right).-The records show the effects of movements of the stimulating or recording electrodes. Record (A) shows the responses recorded when the stimulating cathode was over the median nerve at the wrist and the recording electrodes over the nerve above the elbow. (B) shows the result of moving the stimulating cathode from over the nerve to the radial styloid, keeping the other conditions as in (A). (C) shows the result of leaving the cathode over the nerve at the wrist but moving the recording electrodes medially off the nerve on to the belly of the biceps muscle. The time scales show 0.1 and $1 \mathrm{msec}$. intervals. 


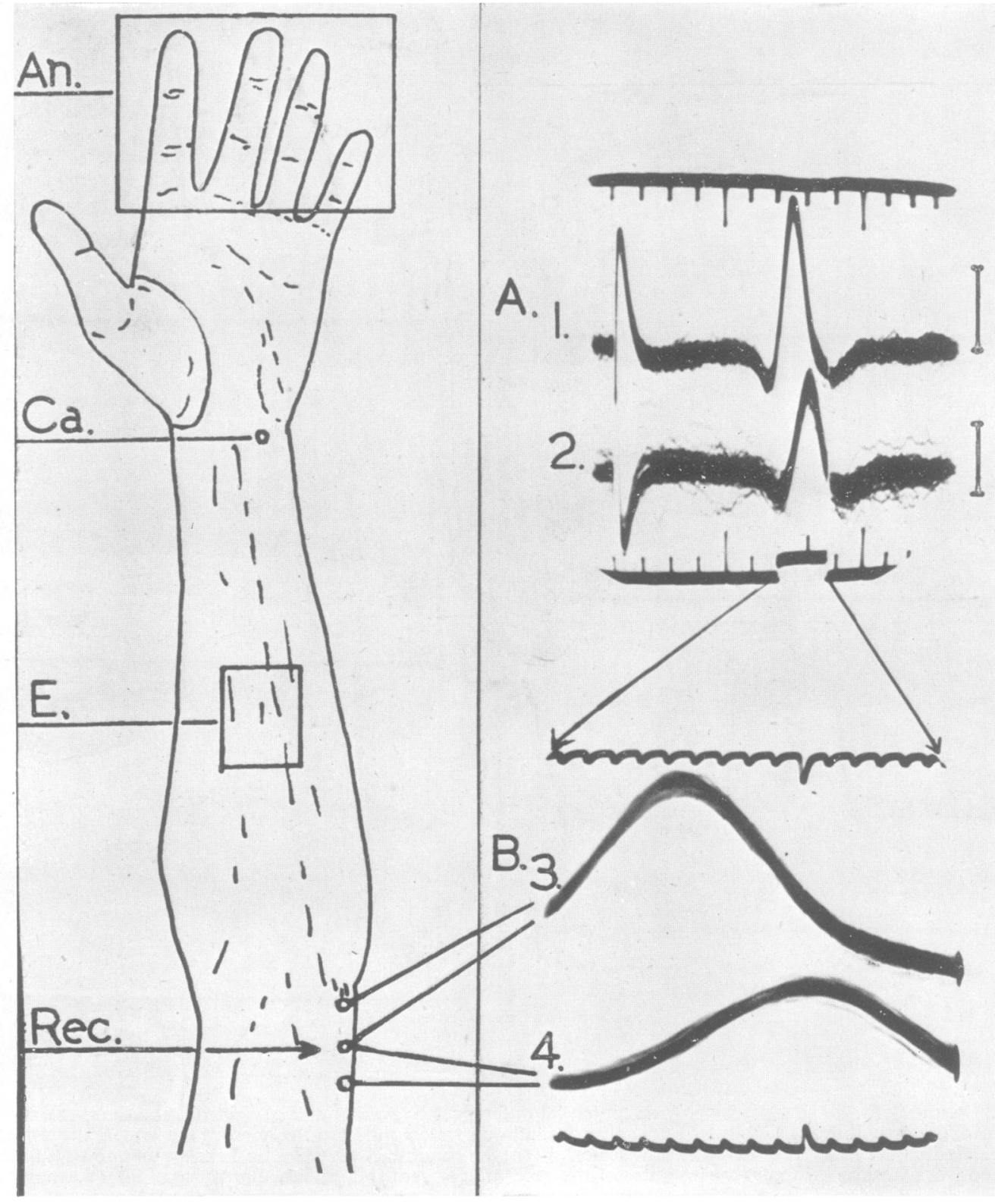

Fig. 4.-The records show the change in the latency of the responses with change in distance from the stimulating point. Traces 1 and 3 were recorded simultaneously from the distal and central electrodes, traces 2 and 4 from the central and proximal ones. The response from the proximal pair of electrodes is delayed by 0.5 msec. In the diagram the letters indicate : An., anode and Ca., cathode of the stimulator, E, earth connexion, Rec., recording electrodes separated by $3 \mathrm{~cm}$. The time scales in (A) show interv. Is of 1 and 5 msec. and in (B) of $0.1 \mathrm{msec}$. The calibration marks in (A) indicate $20 \mu \mathrm{V}$. 

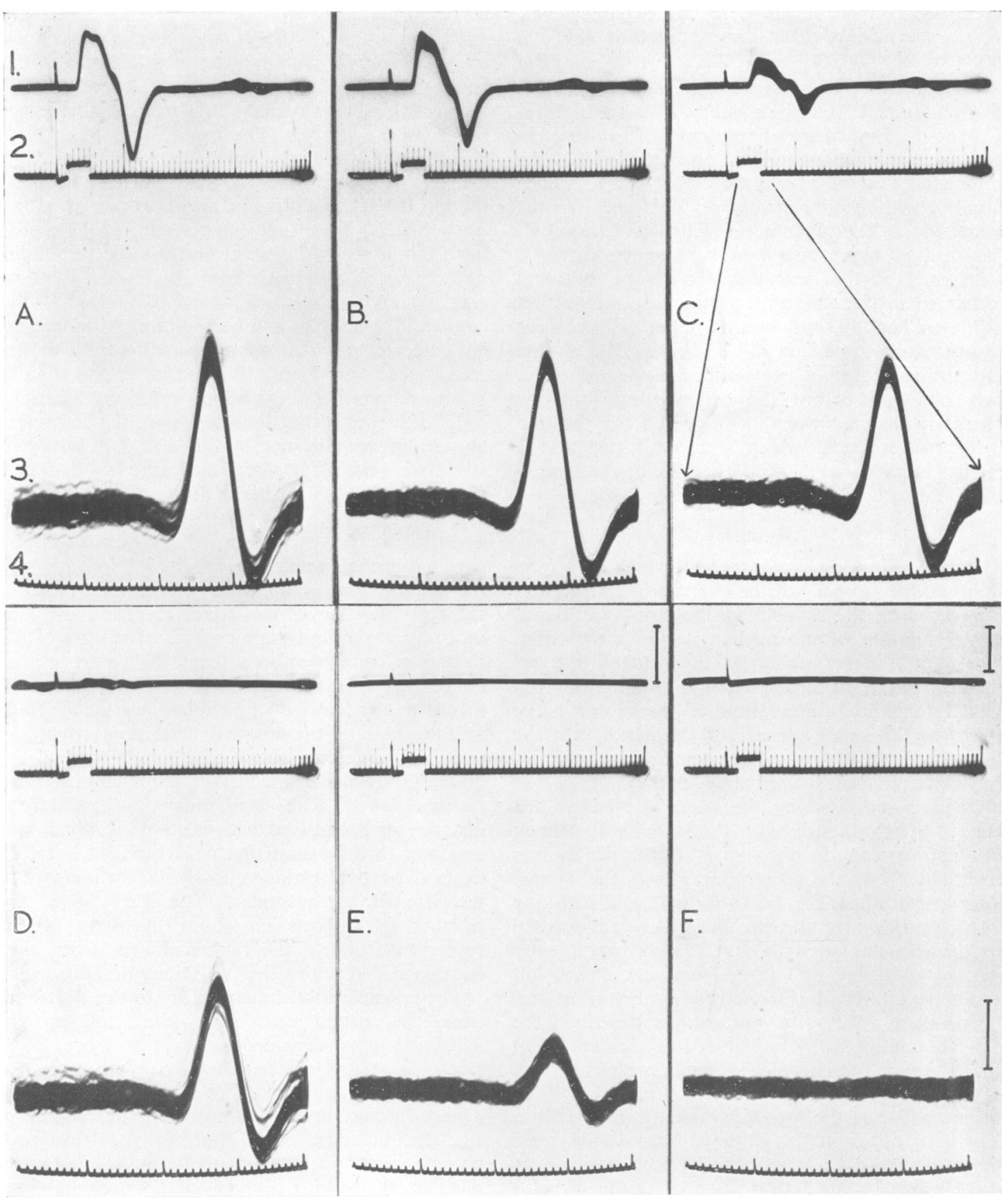

FIG. 5.-The records show the relation between the action potential volley in the median nerve and the number of motor fibres being stimulated. In trace 1 are recorded the action potentials of the muscles in the thenar eminence, and in trace 3 the action potentials from the median nerve above the elbow. In (A) the stimulus to the nerve at the wrist was supramaximal for the motor fibres, and in (B), (C), (D), (E), and (F), the strength of the stimulus was progressively reduced. Records (D) and (E) show that the greater part of the nerve action potential in (A) is due to fibres with a lower threshold than motor fibres. Trace 2 shows the time scale for trace 1 in 1,5 , and $20 \mathrm{msec}$. intervals, and the pedestal shows the relative time and duration of trace 3 , the time scale for which is shown in intervals of 0.1 and $1 \mathrm{msec}$. in trace 4 . The calibration mark in (E) at the end of trace 1 shows $8 \mathrm{mV}$. for records (A) to (E), and that in (F) $400 \mu \mathrm{V}$. The mark at the end of trace 3 in (F) shows $20 \mu \mathrm{V}$. for this trace in all the records. 
was taken to cathode ray tubes from which photographic records were made of either single responses or up to fifty responses superimposed.

Using the methods described, fifteen subjects have been examined. Of these subjects, thirteen could give no history of any neurological disorder ; in the fourteenth the ulnar nerve on one side had been completely severed above the elbow and had been sutured, with good recovery of function. On the same side as the ulnar nerve which had been cut, the median nerve also had been damaged by a tourniquet at the operation for suture, but had recovered in three months. Otherwise this subject also was healthy, and in this paper only records from the uninjured side will be considered. In the fifteenth subject no history of neurological disorder was obtained before the examination, but the results in his case were so different from those in the other subjects, which were uniform, that a further inquiry was made. This disclosed a history of mild disorder which will be discussed later.

\section{Results}

Fig. 2A shows an oscillogram in which are superimposed fifty records of the changes of potential at electrodes on the skin above the elbow, produced by stimulation of the median nerve at the wrist. The stimuli were applied at the rate of one per second and were strong enough to give approximately maximal stimulation of the motor nerve fibres to the small muscles of the hand. On the lower trace in the record are shown time intervals of 1 msec., and the instant at which the stimulus was applied is indicated by the black dot below this trace. Synchronously with the stimulus the shock artefact appears in the upper trace, which was recorded from the electrodes above the elbow. This artefact lasts for 1.5 to $2 \mathrm{msec}$. and with any one electrode arrangement its size is proportional to the stimulus strength, but it may vary greatly in size, duration, and form from one experiment to the next and with the electrode contact resistance or position. With the precautions described the artefact could usually be reduced to such an extent that the baseline became flat again within $2 \mathrm{msec}$. of the stimulus, even when this was of fifty volts or more and when the record was being made with a sensitivity of $1 \mathrm{~mm}$. deflection per microvolt between the recording electrodes. Next there is a triphasic wave which begins 3 to $4 \mathrm{msec}$. after the stimulus and which is complete in a further $3 \mathrm{msec}$. When the stimulus was increased slowly from zero the stimulus artefact appeared first, then at a threshold level, which was accurately repeatable, the triphasic wave appeared. This wave grew rapidly up to a point where an increase of stimulus strength of
20 or 30 per cent. produced no significant increase in its size, although the stimulus artefact continued to get larger. The stimulus strength at which the of wave first ceased to increase was approximately $\overrightarrow{0}$ that which gave maximal stimulation of the motor $D$ fibres in the nerve. The example in Fig. 2A is typical of the records which were obtained from over the median or ulnar nerves of fourteen subjects, though the relative sizes of the three phases of the wave varied from subject to subject. Records from the fifteenth subject at first showed no similar deflections at all, apart from the usual stimulus artefact. At a later examination, and after the courses of the nerves had been plotted with particu- $\bar{\alpha}$ lar care, regular deflections were detected in the records between 4 and $10 \mathrm{msec}$. after the stimuli. When stimulating the median nerve on the right $\vec{O}$ or left side these deflections were only just detectable above the irregularities in the records, and when ${ }_{\sigma}$ stimulating the ulnar nerves they were less than half the size of the deflections in any of the other fourteen subjects. The small size of the responses $\vec{N}$ and the limited area over which they could be ${ }_{A}^{N}$ detected probably accounts for the failure to record i them at the first examination. In the other fourtee 0 subjects the responses were recorded witholf difficulty at the first examination. The sizes of the responses were measured from the centre of the $z$ darkest part of the baseline in the superimposed record to the centre of the darkest part of the trae 3 at the peak of the upward deflection, when the potential difference between the electrodes w greatest. The measurements were all made from records taken with a standard inter-electrode distance of $3 \mathrm{~cm}$. and with a stimulus which was maximal to the extent that a 20 per cent. increase in its size would produce no significant increase in the size of the response. The Table gives the number of subjects in whom the sizes of the $\mathbb{\mathscr { Q }}$ responses fell into the ranges shown, when they $\overrightarrow{\vec{\sigma}}$ were recorded under the conditions described.

The connexion of the amplifiers to the electrodes over the nerve was such that an upward deflection was recorded when the more distal electrode of a pair became negative with respect to the more proximal one. The deflections between 3 and 7 msec. after the stimuli in the records in 3 . Fig. 2A, therefore, show that the distal electrode $\delta$ became first positive, then negative, and then positive again with respect to the proximal one. 응 This triphasic form appeared constantly when the electrodes were at places where the nerve could be stimulated equally easily, and where it was probably equally superficial. Occasionally, when a pair of $\mathbb{N}$ electrodes over the median nerve was more distal N than usual, a predominantly diphasic wave was N 
TABLE

RANGE OF SIZES OF RESPONSES RECORDED

\begin{tabular}{c|l}
\hline $\begin{array}{c}\text { No. of } \\
\text { Subjects }\end{array}$ & \multicolumn{1}{|c}{$\begin{array}{c}\text { Amplitude of responses : } \\
\text { microvolts }\end{array}$} \\
\hline 1 & less than 10 \\
\hline 1 & from 10 to less than 20 \\
\hline 5 & from 20 to less than 30 \\
\hline 2 & from 30 to less than 40 \\
\hline 3 & from 40 to less than 50 \\
\hline 3 & from 50 up to 60 \\
\hline 0 & more than 60 \\
\hline
\end{tabular}

recorded. This may have been due to the distal electrode being over the nerve where it was becoming less superficial as it entered the antecubital fossa. In these circumstances a typical triphasic wave was still recorded from a pair of electrodes farther up the arm. Before it may be accepted that these or similar changes are due to nerve action potentials certain sources of artefact must be excluded.

From the fifty superimposed records in Fig. 2A it seems that the triphasic wave follows each stimulus by an interval. which varies by not more than plus or minus $0.1 \mathrm{msec}$. Disturbances not related to the stimulus, such as action potentials associated with steady or random contractions of muscles near to the recording electrodes, produce only irregular deflections at different parts of the trace. In Fig. 2B are shown fifty superimposed records made from the same electrodes and under the same conditions as the records in Fig. 2A, except that no stimulus was applied to the nerve. No deflections appear like those in the records in Fig. 2A. This suggests that the disturbance was provoked by the stimulus and that the time at which it occurred was accurately related to the time of the stimulus. The records in Fig. 3 show the effects of movements of the stimulating or recording electrodes away from the nerve. Fig. 3A shows the responses recorded when the stimulating cathode was over the median nerve at the wrist and the recording electrodes were over the nerve above the elbow. In Fig. 3B the records show the result of moving the stimulating cathode away from the nerve to a point over the styloid process of the radius. The stimulus artefact in the record was almost unaltered but the later deflections disappeared completely. This suggests that these later deflections were not due to spread of the stimulating current, or to any of its effects on tissues other than the nerve. When the stimulus was applied to the nerve, the small muscles of the hand which were innervated by the particular nerve being stimulated, twitched with, each stimulus and their action potentials were about $8 \mathrm{mV}$. in amplitude from the baseline to the first peak. The latency between a stimulus to the median nerve at the wrist and the beginning of the action potentials in the thenar muscles was between 4 and 5 msec., which was very similar to the latency between the stimulus and the beginning of the response at the electrodes above the elbow. The disturbance above the elbow, which was never more than $60 \mu \mathrm{V}$. in size, might therefore have been an electrical spread of the action potentials from the twitching muscles in the hand. If the recording electrodes were moved away from the nerve, on to the belly of the biceps muscle, the size of the recorded deflections was reduced or they were abolished. The records in Fig. 3c show this effect. There is greater irregularity in the baseline because of incomplete relaxation of the biceps muscle, and the deflections $4 \mathrm{msec}$. after the stimulus are much smaller than in the records in Fig. 3A. They were probably not abolished in this experiment because the recording electrodes were of the rectangular strip type and to abolish the deflections it was necessary to move them two and a half to three times as far from the nerve as when circular electrodes, $1 \mathrm{~cm}$. in diameter, were used.

The type of experiment described showed that the potential changes were greatest over the plotted course of the nerve ; if they were due to electrical conduction of the action potentials from the hand muscles there was no reason why this should have been so. In addition the action potentials from the thenar muscles were some five times longer in duration than the potential changes recorded from the electrodes above the elbow. The reduction in size of the response as the electrodes were moved away from the nerve and on to the biceps muscle also suggests that it was not due to a reflex twitch of the biceps muscle caused by the shock, and this is supported by the short latency of only 3 to $4 \mathrm{msec}$. between the stimulus and the response. Additional and .confirmatory evidence that the disturbance was being conducted in nerve was found in measurements of the latency of the response at different distances from the stimulating electrodes. Fig. 4 shows two records made simultaneously from three electrodes at intervals of $3 \mathrm{~cm}$, with the arrangement indicated in the diagram. In Fig. 4 the response from the distal electrode and the central one, traces 1 and 3 , occurred earlier than that from the central electrode and the proximal one, traces 2 and 4 . The latencies of the negative peaks of the waves differed by $0.5 \mathrm{msec}$. This is 
shown in Fig. 4B, where these peaks were displayed on an expanded time scale the duration of which corresponded to the pedestal in the lower time scale in Fig. 4A. There may be considerable errors in estimations of conduction velocity made from records of this type, where the sizes of the two waves are not identical, but a time of $0.5 \mathrm{msec}$. for a conduction distance of $3 \mathrm{~cm}$. would indicate a conduction velocity of the same order as that in motor nerve fibres in man. Certainly the occurrence of an initial positive wave at the electrode nearer to the point of stimulation does not conflict with the idea that the changes were due to a volley of impulses ascending the nerve towards the recording electrodes. When the region of relative negativity associated with a volley of nerve impulses passes along a nerve which is in a large volume of conducting medium, a similar triphasic variation of potential difference appears between an electrode near to the nerve and one remote from it. The electrode nearer to the nerve becomes first positive with respect to the other, then negative, and finally positive again. A full analysis of this sequence of events has been made (Lorente de No, 1947), which applies to the present recording arrangements even though in these the two electrodes were both relatively close to the nerve and at the boundary of the conducting medium.

Since the findings described above indicated that the changes at the recording electrodes were due to nerve action potentials, and were not due to artefact, experiments were made to determine the type of nerve fibres concerned. The strength of stimulation that was used suggested that the fibres being stimulated had a similar excitability to that of the motor fibres. Stimulation of the motor fibres will produce an ascending as well as a descending volley of impulses, so part or all of the action potentials recorded at the elbow might have been due to these antidromic impulses in motor fibres. The experiment illustrated in Fig. 5 was made to test this point. In it the median nerve was stimulated at the wrist, the action potentials in the muscles of the thenar eminence were recorded from surface electrodes, and fifty records were superimposed. Trace 1, at the top of the records, shows the muscle action potentials, and trace 2 shows time intervals of 1,5 , and $20 \mathrm{msec}$. and the first big spike indicates the time of stimulation. Trace 3 is a record of the action potentials in the nerve above the elbow, and trace 4 shows the time scale for this record with intervals of $0 \cdot 1$ and $1 \mathrm{msec}$. The time relation of traces 3 and 4 to that of traces 1 and 2 is shown by the pedestal in trace 2 . In Fig. $5 \mathrm{~A}$ the stimulus used was maximal for the motor fibres to the thenar muscles. In the records in (B), (C), (D), (E), and (F), the strength of stimulus was progressively reduced. In record (D), where the stimulus was only just aboveo threshold for the motor fibres, and when no muscular? twitching could be seen elsewhere, the nerve action potential volley has only declined to 70 per cent. 00 of its original size. This suggests that the greatero part of the volley recorded in (A) was not in motor fibres but in other fibres which had a lower threshold than that of the motor fibres. In this experiment $\Rightarrow$ the subject reported that, with the strengths of stimulus used for records (D) and (E), considerableo sensation was produced in the distribution of the $\underline{\bar{E}}$ median nerve in the fingers. This indicates that $\frac{\bar{p}}{\frac{p}{}}$ where the muscle action potential which was $\mathbb{\complement}$ produced by the stimulus used in (A) had disappearedo and no evidence of it could be obtained, even with" an increased amplification, such as was used in ${ }^{\circ}$ record (F), sensory afferent fibres other than those $\vec{\omega}$ from muscle were being stimulated. In (F), whereo the nerve action potential also had virtually disappeared, some sensation was still reported by the? subject after each stimulus. Therefore, of the fibres $\vec{\sim}$ in the median nerve which were stimulated and which $\rightarrow$ had a lower threshold than the motor fibres, somen at least were sensory afferent fibres from the finge 0 The proportion of afferent fibres from muscles $\oplus_{0}$ ? those from elsewhere was not determined. in interpreting observations such as these it is necess to consider the division of the nerve into funicudi $\$$ which has been investigated by Sunderland (194), and which makes it possible with weak stimuli $f \circ$ excite alone either motor responses from the thenan muscles or sensations from the fingers, by varyingto slightly the site of stimulation. In the experiments described the stimulating point was chosen to giveo the greatest possible motor responses with weaks stimuli, and therefore the interpretation of the results is probably not,invalidated by any effects of division of the nerve into funiculi.

\section{Discussion}

From the number of observations made, it is not possible to draw any useful conclusions about the. relation of the amount of subcutaneous fat in the subjects examined to the size of the action potentialso which could be recorded. However, the range of build in these subjects was such that it seems reasonable to expect that action potentials of $20 \mu \mathrm{V} .0$ amplitude or more may be recordable under the conditions described in any healthy person. The subject in whom all the action potentials were under $>$ $10 \mu \mathrm{V}$. and in whose median nerves they were barely? detectable had complained some years ago of an patchy anæsthesia and numbness of the left hand which persisted for several months. At that time he was the subject of experiments in which 
frequent readings of blood pressure were made and where in some instances cuffs inflated above systolic blood pressure were applied to either arm for considerable periods. At the time of the present examination there were no obvious residual defects in power or sensation, and it seems that if the nerves were damaged in the earlier experiments the recording of nerve action potentials may be a delicate means of detecting minor degrees of damage. The action potentials recorded from the cut and sutured ulnar nerve and from the median nerve known to have been paralysed by the tourniquet are to be described elsewhere but they also suggest that the method may have value in demonstrating small degrees of damage to nerve.

One point of interest has been the apparent homogeneity of the group of fibres being examined by the methods described. The action potential was conducted over a distance of 25 to $30 \mathrm{~cm}$., and might have been expected to show more temporal dispersion than appears in the records. The fact that so little temporal dispersion appears is probably due to the triphasic shape of the recorded wave, since this may have masked later deflections. This is suggested by the fact that, in the few records taken, at the expense of considerable discomfort to the subject, with shocks increased slowly up to 50 and 100 per cent. stronger than those used in the other experiments, the first change in the records which appeared was a reduction in the size of the third phase of the wave and later the appearance of quite clear additional deflections at that time.

Attempts to measure the conduction velocity in the ulnar and median nerves with the methods described have proved on the whole unsatisfactory, on account of the difficulty of making pairs of records which have the same shape from electrodes at different positions on the nerve. Using pairs of records which were nearly superimposable, and timing from the start of the stimulus escape to the first reversal of potential difference between each pair of electrodes, gave differences in latency from which the calculated conduction velocities were in no case outside the range described by Helmholtz and Baxt (1870) for motor nerve fibres in the arm in man. These writers also showed that temperature was a more important factor in producing variation in their results than errors in measurement of either time or distance. It seems, therefore, that no more accurate results are likely to be obtained than those they give without accurate control of temperature, which was not made in these experiments.

\section{Summary}

1. In fifteen healthy subjects action potentials have been recorded from electrodes on the skin over the course of the median or ulnar nerve after electrical stimulation of the nerve at the wrist.

2. Sources of artefact are considered, and the methods used for stimulating the nerves and recording the action potentials are described.

3. The type of the fibres from which action potentials may be recorded is considered.

4. It is suggested that the method may have use in the study of minor degrees of nerve injury.

The authors would like to express their gratitude to Dr. E. A. Carmichael, in whose department this work was done. The work was carried out whilst one of us (J.W.S.) was holding a Nuffield Foundation Dominion Medical Travelling Fellowship.

RefERENCES
Dawson, G. D. (1947). J. Neurol. Neurosurg. Psychiat., 10, 134.

Helmholtz, H., and Baxt, N. (1870). Mber. Kgl. Preuss. Akad. Wissenschaften, Berlin. p. 184.

Lorente de No (1947). Studies of the Rockefeller Institute, vol. 132, chap. 161, p. 384.

Rusinov, V. S. (1943). C. R. (Doklady) Acad. Sci. U.R.S.S., $41,36$.

(1947). Amer. Rev. Soviet Med., 4, 436.

- , and Chugunov, S. A. (1939). Bull. biol. med. exper. U.R.S.S., 8, 70.

(1940). Soviet Psikhonevrol., 16, 53.

Sunderland, S. (1945). Brain, 68, 243.

Walter, W. Grey, and Ritchie, A. (1945). Electronic Engineering, 17, 585.

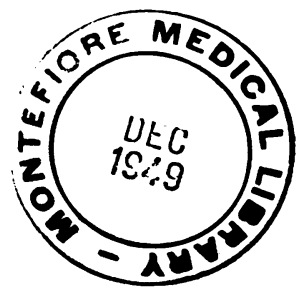

